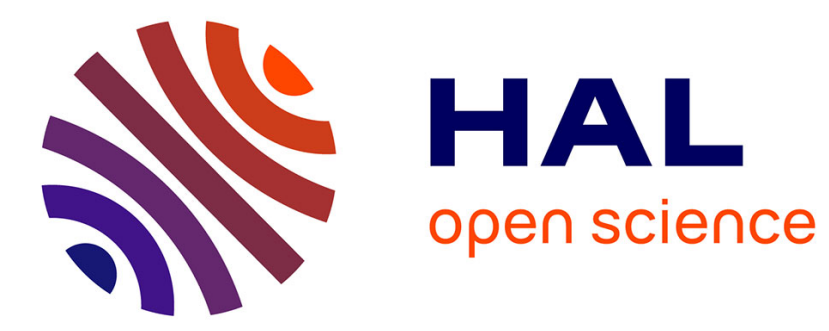

\title{
Conserved scalar mixing in a confined-opposed-jets flow
}

\author{
J.-F. Krawczynski, A. Bouha, B Renou, L Danaila
}

\section{To cite this version:}

J.-F. Krawczynski, A. Bouha, B Renou, L Danaila. Conserved scalar mixing in a confined-opposedjets flow. Journal of Turbulence, 2015, 16 (12), pp.1139-1160. 10.1080/14685248.2015.1066012 . hal-01429845

\section{HAL Id: hal-01429845 \\ https: / hal.sorbonne-universite.fr/hal-01429845}

Submitted on 9 Jan 2017

HAL is a multi-disciplinary open access archive for the deposit and dissemination of scientific research documents, whether they are published or not. The documents may come from teaching and research institutions in France or abroad, or from public or private research centers.
L'archive ouverte pluridisciplinaire HAL, est destinée au dépôt et à la diffusion de documents scientifiques de niveau recherche, publiés ou non, émanant des établissements d'enseignement et de recherche français ou étrangers, des laboratoires publics ou privés. 
Journal of Turbulence

Vol. 00, No. 00, 2015, 1-

\title{
RESEARCH ARTICLE
}

\section{Conserved scalar mixing in a confined-opposed-jets flow}

\author{
J.-F. Krawczynski ${ }^{1,2}$, A. Bouha ${ }^{3}$, B. Renou ${ }^{3}$ and L. Danaila ${ }^{3, *}$ \\ ${ }^{1}$ Sorbonne Universités, UPMC Univ Paris 06, UMR 7190, Institut Jean Le Rond d'Alembert, F-75005, \\ Paris, France \\ ${ }^{2}$ CNRS, UMR 7190, Institut Jean Le Rond d'Alembert, F-75005, Paris, France \\ ${ }^{3}$ CORIA-UMR 6614-Normandie Université CNRS-Université et INSA de Rouen \\ Campus Universitaire du Madrillet 76800 Saint Etienne du Rouvray, France. \\ (Received 00 Month 200x; final version received 00 Month 200x)
}

\begin{abstract}
The focus of this paper is on the mixing of a conserved passive scalar for $S c=1$ ( $S c$ is the Schmidt number) in axisymmetric turbulence for which the initial injections of turbulent kinetic energy and scalar variance are similar. Two confined-opposed-jets (COJ) are experimentally studied through simultaneous PIV (Particle Image Velocimetry) and PLIF (Planar Laser Induced Fluorescence) measurements, for different flow regimes. One-point transport equation for the scalar variance is assessed through experimental data, along the common axis of the two opposed jets, and different physical phenomena are revealed (production, diffusion, dissipation). The production of scalar variance is equilibrated by the diffusion term $(\sim 75 \%)$ and the mean dissipation of the scalar variance $\sim 25 \%$. To further assess the scalar behaviour at each scale in this anisotropic, but axisymmetric, flow, a scale-by-scale scalar variance budget equation is derived for axisymmetric turbulence. This equation reduces to Yaglom's 4/3 law, under additional restrictions. The equation is assessed through experimental data, in the impingement region between the two confined-opposed-jets. In particular, the anisotropic energy transfer along different directions is quantified. It is shown that for scales smaller than the size of the central region, $\Delta$, the cascade of the scalar variance is completely inhibited, independently of the particular direction. For scales larger than $\Delta$, the apparent aspect of the energy transfer is that of an inverse cascade, with positive values of the scalar variance transfer. Nonetheless, inhomogeneity of the flow and mixing at those scales is directly responsible for these positive values.
\end{abstract}

\section{Introduction}

The simplest exact way to analytically describe the behaviour of a passive scalar, $\zeta$, diffusing in a turbulent flow which is randomized through the action of the non-linear advection process is to study the statistical properties of its increments, $\delta \zeta$. This was first developed by A.M. Yaglom [?] who established the relationship between the second-order moment of the scalar increment, $\left\langle(\delta \zeta)^{2}\right\rangle$ and the thirdorder mixed moment, $\left\langle\delta u_{l}(\delta \zeta)^{2}\right\rangle$ in the context of isotropic turbulence

$$
-\left\langle\delta u_{l}(\delta \zeta)^{2}\right\rangle+2 \kappa \frac{d}{d r}\left\langle(\delta \zeta)^{2}\right\rangle=\frac{4}{3}\langle\chi\rangle r
$$

where angular brackets denote time averaging, $\delta u_{l}$ is the longitudinal velocity increment, $\kappa$ is the molecular diffusivity, $\langle\chi\rangle=\kappa\left\langle\partial_{i} \zeta \partial_{i} \zeta\right\rangle$ is the mean scalar dissipation rate, and $\partial_{i} \zeta=\partial \zeta / \partial x_{i}(i=1,2,3)$ is the spatial derivative of $\zeta$ in the $i$ th direction. Equation (??) is of crucial importance for turbulence research, being the only relation which is directly deduced from the scalar transport equation. Equation (??) should be satisfied in the inertial-range scales of any flow, irrespectively of the

\footnotetext{
* Corresponding author: danaila@coria.fr
} 
large-scale properties or the energy injection modes, given the Reynolds numbers are sufficiently high [?]. The balance between the left-hand and right-hand sides of Eq. (??) was, for example, favourably tested against experimental data for small and intermediate scales in a turbulent plane jet [?] or in the counter-rotating, Von Kármán swirling flow at a Taylor Reynolds number $R_{\lambda} \approx 400$ [?].

However, for moderate Reynolds numbers, Yaglom's equation is only valid over a restricted range of scales. When local isotropy holds, large-scale effects are to be taken into account as, for example, the large-scale inhomogeneity in grid turbulence in which the scalar is introduced by a mandoline [? ] and, for a passive scalar injected by a mean scalar gradient in a homogeneous and isotropic turbulent velocity field [?]. Generalized forms of Yaglom's equation were derived to account for the large-scale effects which satisfactorily improved the balance over a significant range (intermediate to large) of scales. Clearly, it is important to identify and quantify the terms that allow the energy balance to be closed, in order to better understand all the physical phenomena brought into play in a region of a particular flow. This statement is even more crucial in the context devoted to understanding and modelling real flows, whereas most of them are anisotropic, at least over a range of scales, and/or populated by coherent structures, the latter being often the illustration of the permanence of the initial/boundary conditions.

The aim of this work is to propose and to use an extension of Yaglom's equation that accounts for anisotropy. This equation is derived in the specific axisymmetric context, and is the analogue to the Yaglom-like equation developed for the kinetic energy at a given scale in axisymmetric turbulence [?]. This analytical tool serves to quantify, at least over a range of scales, the transfer of the scalar energy in anisotropic flows, in tight connection with its production, diffusion and dissipation. The equation will be partially validated in the impingement region among two confined-opposed-jets (hereafter, COJ), in which the quantification of anisotropic energy transfer along different directions will be discussed.

A reduced form of the Yaglom-like equation derived by Danaila et al. [? ] was already tested against experimental data, in the same flow composed of opposed jets. Particular to this flow is that 16 pairs of closely-spaced opposed-jets coexist and interact with their characteristic instabilities, thus leading to local confinement of the basic, periodic pattern. As a result of collisions between confined opposed streams and their subsequent instabilities, a relatively narrow zone of high turbulent intensity is generated, which offers a priori excellent conditions for intensifying mixing rate. This intensification is due to several effects: an increase of the relative velocity between each two opposed streams, an increase of the mean residence time of the fluid in the system (hold-up due to the penetration into the opposed stream), an increase of the interface surface of the fluids coming from two opposed/neighboured jets by the above mentioned instabilities. Although the production of energy which takes place in the impingement region of two-opposed jets from the intense compressive strain of the mean field results in strong anisotropic distribution of energy, the particular geometrical features of this flow prescribes global axisymmetry about the jets axis.

The roadmap of the paper is as follows. The experimental set-up as well as the performed experiments are described in Section ??. The instantaneous and mean values of the conserved scalar are presented in Section ??. The terms involved in one-point budget of the scalar variance are assessed through experimental data in Section ??, with a particular attention paid to the mean dissipation rate of the scalar variance (subsection ??). The core of the paper is Section ?? in which we first derive the transport equation, then some terms (in particular, the energy transferred at each scale, along two particular directions) are evaluated from the 
experimental data. We conclude in Section ??.

\section{Apparatus and measurements}

The experiments were conducted in the confined-opposed-jets chamber, Fig. ??, in which the work of [?] was previously carried out. The test section consisted of a rectangular parallelepiped $\left(110 \times 110 \times 60 \mathrm{~mm}^{3}\right)$ equipped with quartz Suprasil windows $\left(100 \times 80 \mathrm{~mm}^{2}\right)$ on each side. Both the inlet and the outlet for the fluid were located on the top and bottom boundary plates made of a $24 \mathrm{~mm}$-spacing square-matrix arrangement of jets and of a porous, homogeneous, media in between them. The porous boundary plates were backed by plena connected to the exhaust piping network. $200 \mathrm{~mm}$-long tubes were supplying the 16 pairs of jets issuing from the top and bottom boundary plates. Air and air doped with $4 \%$ by volume of acetone are alternatively injected and mixed in the chamber. Thus, the scalar investigated in this paper is the dimensionless mixing fraction. Air mass flow-rates were controlled by two Bronkhorst (EL-FLOW) mass flow-meters whose accuracy was estimated at $\pm 0.5 \%$ of the full scale $\left(100 \mathrm{Nm}^{3} / \mathrm{h}\right)$. Micrometric valves were used to match the flow rate issuing from every individual jet operating at once. The inner-chamber static pressure as well as the pressure drop of each exhaust plena could be adjusted with two regulating valves connected to the exhaust piping network. One wall was equipped with a $1 \mathrm{~mm}$-diameter pressure tap, connected to a digital manometer. A static pressure transducer (Keller) was used to measure the mean flow pressure with a resolution of 1 mbar and and accuracy of 0.03 bars.

The geometry of the facility is characterized by the following outer scales: the horizontal mid-distance between two consecutive jet-axes, $L=12 \mathrm{~mm}$, the vertical mid-distance between two opposed jets, $h=30 \mathrm{~mm}$ and the inner jet diameter, $d_{j}=10 \mathrm{~mm}$ or $d_{j}=6 \mathrm{~mm}$. Consequently, opposed jets meet together at a distance equal to $3 d_{j}$ or $5 d_{j}$ and they are laterally distanced by $2.4 d_{j}$ or $4 d_{j}$.

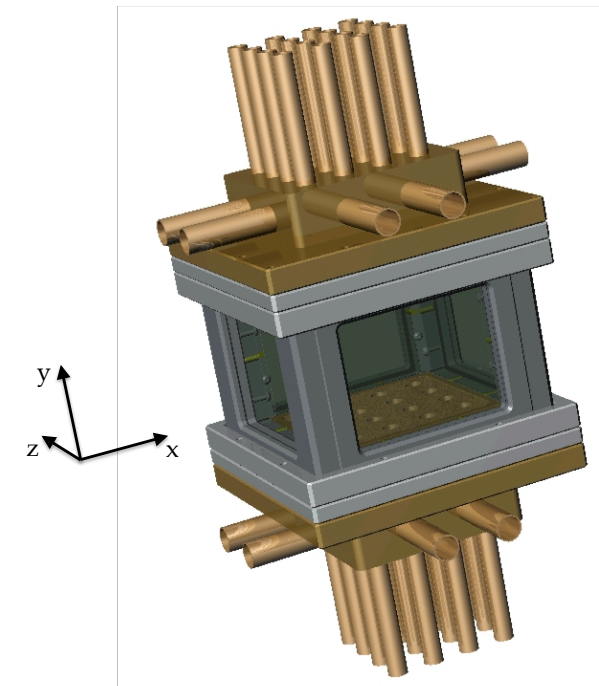

Figure 1. Experimental set-up.

All the experiments conditions whose results are shown in this paper are documented in Table ??, in which we report the flow rate $Q_{v}$, the residence time 


\begin{tabular}{|c|c|c|c|c|c|c|c|c|}
\hline Case & $\mathrm{A}$ & $\mathrm{B}$ & $\mathrm{C}$ & $\mathrm{D}$ & $\mathrm{E}$ & $\mathrm{F}$ & $\mathrm{G}$ & $\mathrm{H}$ \\
\hline$d_{j}[\mathrm{~mm}]$ & 10 & 10 & 6 & 10 & 10 & 6 & 6 & 6 \\
\hline$h / d_{j}$ & 3 & 3 & 5 & 3 & 3 & 5 & 5 & 5 \\
\hline$L / d_{j}$ & 1.2 & 1.2 & 2 & 1.2 & 1.2 & 2 & 2 & 2 \\
\hline$Q_{v}\left[\mathrm{~m}^{3} / \mathrm{h}\right]$ & 63 & 84 & 64 & 136 & 160 & 102 & 139 & 163 \\
\hline$V_{\text {inj }}[\mathrm{m} / \mathrm{s}]$ & 7. & 9.3 & 19.6 & 15. & 17.7 & 31.4 & 42.8 & 50.3 \\
\hline$\tau_{\mathrm{R}}[\mathrm{ms}]$ & 41.2 & 31 & 41 & 19.2 & 16.3 & 25.5 & 18.7 & 16 \\
\hline$Q_{\mathrm{inj}},\left[\mathrm{m}^{4} / \mathrm{s}^{2}\right] \times 10^{-4}$ & 38. & 68. & 109. & 177. & 246. & 279. & 518. & 715. \\
\hline$R e_{\text {inj }} \times 10^{3}$ & 6.4 & 8.5 & 10.7 & 13.7 & 16.25 & 17.3 & 23.5 & 27.7 \\
\hline
\end{tabular}

Table 1. Experimental conditions.

$\tau_{R}$ (defined as the ratio between the chamber volume and the flow rate), the jets momentum flux $M_{0}$ and the injection Reynolds number for each jet. All the measurements were performed at $P=1.4$ bar and at room temperature $T=19 \pm 2^{\circ} \mathrm{C}$. The kinematic viscosity of the air was $\nu=1.08910^{-5} \mathrm{~m}^{2} / \mathrm{s}$ (the error due to the temperature variations is $\pm 2 \%$ ). Except at the lowest velocities, injection is at highenough values of $R e_{\text {inj }}$ to be fully turbulent [?]. This facility guarantees stationary boundary conditions and its geometry, though complex, imposes its symmetries to the mean flow. Statistics discussed in this paper are based on time-averaging performed in each image pixel over a set of 1500 images, which was verified to be large enough to guarantee good convergence of the statistics up to the second-order moments.

\subsection{Velocity field measurements}

The instantaneous, two-dimensional velocity measurements relied on Particle Image Velocimetry (PIV) with DEHS (Di-Ethyl-Hexyl-Sebacat, $\mathrm{C}_{26} \mathrm{H}_{50} \mathrm{O}_{4}$ ) droplets $\left(\rho_{p}=918 \mathrm{~kg} / \mathrm{m}^{3}\right)$. The light source was a Nd-Yag laser (Big Sky laser, $120 \mathrm{~mJ} /$ pulse) with a second-harmonic-generating crystal producing a Q-switched laser output in the green $(532 \mathrm{~nm})$. Light scattered from the droplets was collected on a CCD camera (FlowMaster La Vision, 12 bits, $1280 \times 1024$ pix $^{2}$ ) with a $50 \mathrm{~mm} \mathrm{f} / 1.2$ Nikkor lens. The optical arrangement yielded a magnification of $20.5 \mathrm{pix} / \mathrm{mm}$, calibrated using images of a precision reference grid. PIV processing was performed with a cross-correlation technique between pairs of successive images. The initial size of the PIV interrogation window was $64 \mathrm{pix}^{2}$. Six iterations were used to obtain a final interrogation window size of $16 \mathrm{pix}^{2}$, with a $50 \%$ overlap. The time interval between two consecutive images was in the range $3-8 \mu \mathrm{s}$, scaled approximately inversely with the injection velocity, $V_{i n j}$. The determination of the minimum fully-resolved length-scale was discussed in details in [? ]. An analysis based on the determination of the PIV spectral transfer-function yielded to a cut-off length scale due to the low-pass filtering effect of our PIV system of $\lambda_{c}=1 / k_{c} \simeq 1.7$ $\mathrm{mm}$. The spatial resolution, as well as the largest measurable scales, are fixed for all the experiments by the optics, the CCD focal-plane array resolution, and the PIV processing, and are independent of the flow. The DEHS droplets diameters generated by the seeding apparatus, separately calibrated with a Malvern diffractometer, were in the range $1 \mu \mathrm{m} \leq d_{p} \leq 2 \mu \mathrm{m}$. The corresponding Stokes time was in the range $2.8 \mu \mathrm{s} \leq t_{p} \leq 11.3 \mu \mathrm{s}$ for the room-temperature air $\left(T=19 \pm 2^{\circ} \mathrm{C}\right)$ used as the fluid in these experiments. Characteristic flow times corresponding to a given spatial scale must be larger than particle response time, if droplets are to track the flow at that scale. Flow times that needed to be resolved by the PIV measurements, $t_{c}$ were related to flow structures at the resolution length-scale that were driven by the injection velocity, $\lambda_{c} / V_{i n j}$. Given the highest injection velocity 
in these experiments, $V_{i n j} \simeq 50 \mathrm{~m} / \mathrm{s}$, the minimum Lagrangian time encountered at the resolution length-scale was $t_{c, \min } \simeq 37 \mu \mathrm{s}$. All the droplets were then expected to track resolvable Lagrangian velocity field fluctuations.

\section{2. $\quad$ Scalar field measurements}

Digital imaging of planar laser-induced fluorescence (PLIF) was used to measure mixed-fluid concentration fields in the test section. Excitation of large planar sheets of fluid containing acetone vapours may be readily achieved with a frequencydoubled Q-switched Nd:YAG laser (Continuum YG661) at $\lambda_{L}=266 \mathrm{~nm}$. The laser provided $120 \mathrm{~mJ} /$ pulse (10 pulses/s) with a pulse duration of approximately 8 ns. An optical coloured glass filter BG12 was used to isolate the fluorescence emission from the laser wavelength. Experiments were performed in a darkened laboratory to further minimize noise from ambient light. The effects of laser sheet attenuation were minimized by using a low acetone concentration ( $4 \%$ in volume) in air. In order to illuminate a thin slice of mixed fluid concentration, a laser sheet was formed by the combination of a cylindrical lens and a spherical lens. The sheet thickness given by its FWHMT was of $0.1 \mathrm{~mm}$ in the test section. Images of mixed-fluid concentration field were recorded on an intensified (GEN-II) CCD camera (Princeton PI-MAX:512), capable of 16-bit dynamic range at $(512 \times 512)$ pixel resolution at a framing rate of $10 \mathrm{~Hz}$, with a $50 \mathrm{~mm}$ Nikkor lens $(f: 1 / 1.2)$. The pixel (in-plane) resolution was $0.09 \mathrm{~mm}$, which is comparable to the laser-sheet thickness.

For weak excitation, fluorescence from molecular tracer with broadband absorption such as acetone can be modelled according to [?]

$$
S_{F}(x, y)=I_{0}(x, y, \lambda) d V_{c} \eta_{o p t}\left[\frac{\chi_{\text {Acetone }}(x, y) P}{k T}\right] \sigma(\lambda, T) \Psi\left(\lambda, T, P, \sum_{i} \chi_{i}\right)
$$

where $S_{F}(x, y)$ is the modelled fluorescence signal, $I_{0}(x, y, \lambda)$ is the local laser energy-density in the detection volume $d V_{c}$, and $\eta_{\text {opt }}$ is the overall efficiency of the collection optics. The term in brackets represents the acetone number density, given as the product of mole fraction $\chi_{\text {Acetone }}(x, y)$ and total pressure $P$, divided by the Boltzmann constant $k$ times the temperature $T$. The final two quantities are $\sigma$, the molecular absorption cross-section of the tracer, and $\Psi$ the fluorescence quantum yield. The quantification of the mixed-fluid concentration field of acetone relies on different assumptions. The pressure and the temperature are assumed to be uniform and constant within the test section, the molecular diffusivity is neglected compared to the turbulent diffusivity, and the flow rate does not present any composition variation. Then for a given excitation wavelength, the emitted fluorescence signal is linearly proportional to concentration. This enables quantitative measurement of mixed-fluid concentration field, using the procedure described below. Because of the linearity of fluorescence with concentration, the imaged intensity of fluorescence, $I_{F}(x, y, t)$, of a time-varying concentration field, $c(x, y, t)$, can be written as,

$$
I_{F}(x, y, t)=h_{S}(x, y, t) c(x, y, t)+I_{\text {back }}(x, y, t)+I_{\text {noise }}(x, y, t),
$$

where $h_{S}(x, y, t)$ is an undetermined function of the local laser intensity and the pixel-by-pixel sensitivity of the imaging system. $I_{b a c k}(x, y, t)$ is the cumulative background level due to dark noise, offsets, etc. in the CCD camera. The imaged inten- 
sity of a reference, uniform-concentration field would be,

$$
I_{F, r e f}(x, y, t)=h_{S}(x, y, t) c_{r e f}+I_{b a c k}(x, y, t)+I_{\text {noise }}(x, y, t),
$$

where $c_{r e f}$ is a known concentration. The imaged intensities are contaminated by various noises $I_{\text {noise }}(x, y, t)$, such as the image intensifier multiplication (electron avalanche) noise, the photon shot noise proportional to the square-root of the pixel output value, and the CCD readout noise (electronic noise) from amplifiers onboard the CCD. The image intensifier multiplication noise, proportional to the local/pixel signal and some power of the intensifier gain, is the most important noise in this work and becomes predominant for the fluorescence signal. The laserillumination fields for Eqs. (??) and (??) are assumed to be the same, because the laser attenuation due to absorption is negligible. Then, the mixed-fluid concentration fields were computed by correcting the imaged intensity of fluorescence for the background influence and the laser sheet inhomogeneities,

$$
\begin{aligned}
\frac{c(x, y, t)}{\left\langle c_{r e f}\right\rangle(x, y)} & =\frac{I_{F}(x, y, t)-\left\langle I_{\text {back }}\right\rangle(x, y)}{\left\langle I_{F, r e f}\right\rangle(x, y)-\left\langle I_{\text {back }}\right\rangle(x, y)}+I_{\text {noise }}^{\prime}(x, y, t) \\
& \equiv I_{F, \text { corr }}(x, y, t)+I_{\text {noise }}^{\prime}(x, y, t) .
\end{aligned}
$$

Thus, the ability to make quantitative measurements of scalar concentration using pulsed-laser PLIF measurements only depends on weak absorption and the linearity of fluorescence with concentration, which were both verified for the present experiments.

The correction and normalization procedure described by Eq. (??) only provides a low-frequency correction for the noise contribution. Thus, the mixed fluid concentration field is likely to be influenced by noise, present at small scales, as represented by term $I_{\text {noise }}^{\prime}(x, y, t)$ in Eq. (??). An adaptive Wiener filter [? ? ] was thus developed and applied to every single mixed-fluid concentration field. It is briefly introduced in the following.

Assuming that the noise $I_{\text {noise }}^{\prime}(x, y, t)$ can be modelled as uncorrelated with the local acetone concentration, the spectrum of $\frac{c(x, y, t)}{\left\langle c_{r e f}\right\rangle(x, y)}$, noted $\hat{C}_{c o r r}\left(k_{x}, k_{y}, t\right)$, can be expressed in terms of the spectrum $\hat{I}_{F, \text { corr }}\left(k_{x}, k_{y}, t\right)$ of $I_{F, \text { corr }}(x, y, t)$, and the spectrum $\hat{I}^{\prime}{ }_{\text {noise }}\left(k_{x}, k_{y}, t\right)$ of the noise $I_{\text {noise }}^{\prime}(x, y, t)$,

$$
\widehat{C}_{\text {corr }}\left(k_{x}, k_{y}, t\right)=\widehat{I}_{F, \text { corr }}\left(k_{x}, k_{y}, t\right)+\widehat{I}_{\text {noise }}^{\prime}\left(k_{x}, k_{y}, t\right) .
$$

where $k_{x}$ and $k_{y}$ are the respective wavenumbers in the $\mathbf{x}$ and $\mathbf{y}$ directions. If the noise field and its corresponding spectrum ${\widehat{I^{\prime}}}_{n o i s e}\left(k_{x}, k_{y}, t\right)$ can be directly inferred from the measurements, the spectrum of the mixed fluid concentration field can be determined from Eq. (??) by subtracting $\widehat{I}^{\prime}{ }_{n o i s e}\left(k_{x}, k_{y}, t\right)$ from the spectrum of the corrected imaged intensity of fluorescence $\widehat{I}_{F, c o r r}\left(k_{x}, k_{y}, t\right)[?]$. However, this difference is very sensitive to the accuracy of the noise and fluorescence measurements, especially at high frequencies. Another solution is to derive an optimal Wiener filter [?] such as

$$
\widehat{C}_{\text {corr }}\left(k_{x}, k_{y}, t\right)=\widehat{I}_{F, \operatorname{corr}}\left(k_{x}, k_{y}, t\right) \cdot \Phi\left(k_{x}, k_{y}\right)
$$


with

$$
\Phi\left(k_{x}, k_{y}\right) \equiv \frac{\left|\widehat{I}_{F, c o r r}\left(k_{x}, k_{y}, t\right)\right|^{2}-\left|\widehat{I}_{n o i s e}^{\prime}\left(k_{x}, k_{y}, t\right)\right|^{2}}{\left|\widehat{I}_{F, \text { corr }}\left(k_{x}, k_{y}, t\right)\right|^{2}} .
$$

The interest of this filtering technique is that the cut-off frequency is directly given by the spectral analysis of the initial fluorescence images and consequently adapted to each set of measurements.

Background images were recorded shortly before each run with the laser firing but without starting the seeding. Then, reference images of a uniform concentration field were recorded by feeling the chamber with a homogeneous mixture of air and acetone. Using these background and reference images, the effects of CCD sensitivity variation, illumination non-uniformity, and optical transfer function were removed using Eq. (??). This finally yields the normalized mixed-fluid concentration values

$$
0 \leqslant \zeta(x, y, t) \equiv \frac{c(x, y, t)}{\left\langle c_{r e f}\right\rangle(x, y)} \leqslant 1
$$

Consequently, $\zeta=0$ and $\zeta=1$ correspond to pure unmixed fluids. Any possible shot-to-shot variations in the power of the pulsed laser were corrected over the sheet profile by monitoring the fluorescence-intensity fluctuations in the unperturbed jets potential-cores.

\section{Instantaneous and mean scalar fields}

A detailed description is presented in this section of the $S c \approx 1$ conserved scalar fields $\zeta(x, y, t)$ in the statistically steady, incompressible, confined-opposed-jets flow.

Figure ?? shows typical distributions of the mean scalar concentration field, (a) and the conserved scalar instantaneous fields, (b) and (c), for one pair of opposedjets as a function of $x / d_{j}$ and $y / d_{j}$. The jet centreline coincides with $x / d_{j}=0$. In each case, pure blue denotes the lowest range of scalar values, beginning at $\zeta=0$ (pure air without acetone) and pure red denotes the highest range of scalar values, ending at $\zeta=1$ (air seeded with acetone), with colours ranging from blue to red identifying a linear increase of the scalar concentration values. Velocity field vectors are superimposed to the mixed fluid concentration fields to help with the analysis.

A first comment to be done is about the symmetry of this flow which is remarkably illustrated by the global structure of the mean scalar field. The mean position of the stagnation points is easily identifiable. This particular point of the flow is related to a mean value of the scalar field of $Z=0.5$ and a zero mean velocity field, $U_{x}=0$ and $U_{y}=0$. In the following, the origin of our coordinate system, $(x=0, y=0)$ is arbitrary chosen so that it coincides with the mean stagnation point position. It is thus kept fixed for each experimental case.

The well-defined organization of the mean scalar field contrasts with the aspect of the instantaneous scalar field, as illustrated by the instantaneous maps of scalar and velocity fields in Figs. ??b and ??c. This suggests the systematic presence of large-amplitude oscillations of the very thin fluid interface among each two opposed jets, mainly following the axial direction (perpendicular to the mean plane of stagnation). This interface is materialized by a thin layer of instantaneous concentration equal to the average concentration, $\zeta=Z=0.5$. This characteristic feature of the confined-opposed-jets flow was already discussed in detail in a previous work, with 


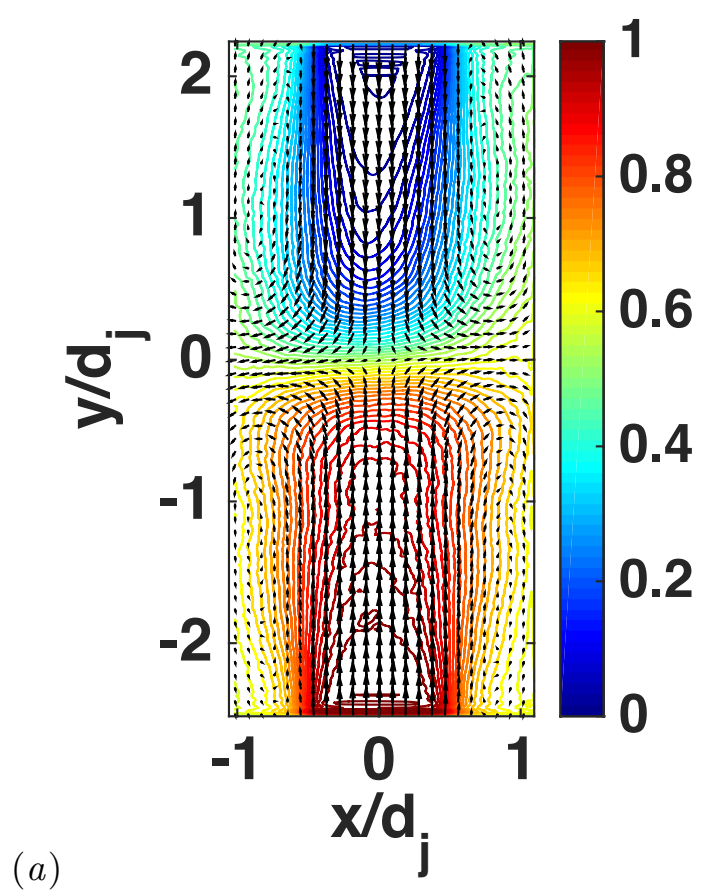

$(b)$
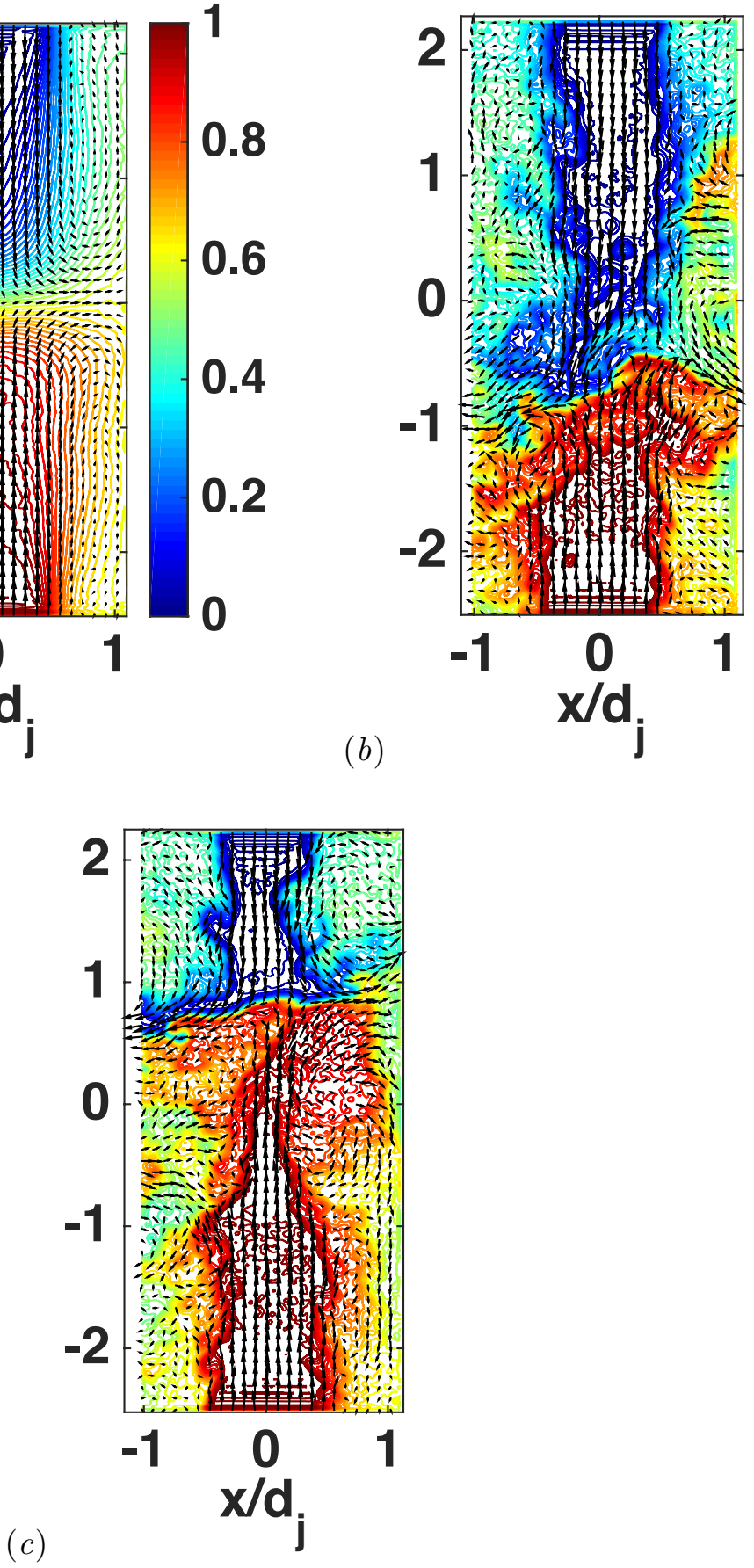

Figure 2. (a): Contour plots of the mean scalar concentration field, $Z$ and normalized mean velocity field, $U / U_{i n j}$, as a function of $x / d_{j}$ and $y / d_{j}$. (b) and (c): Two examples of instantaneous scalar and velocity fields, as functions of $x / d_{j}$ and $y / d_{j}$. Contour plots in increments of 0.02 and bounded by 0 and 1. Displayed are 1 of every 10 vectors. Case A.

a particular insight given to the actual nature of the velocity fluctuations, see [? ] and references therein. Some major features are recalled here. It was shown that the instantaneous interface oscillation was a random process which is characterized by a standard deviation, $\Delta$, that corresponds to the characteristic length scale over which the mean velocity decays to zero in between two confined-opposed-jets. The width over which the mean velocity decreases in between two confined opposed jets may thus be seen as the result of the temporal averaging of the thin instantaneous 
interface "fluctuation" contained in a range of $\pm \Delta$ thick around the mean plane of stagnation.

The presence of vortices at the interface of two opposed jets is also highlighted. This type of structure, to our knowledge first evidenced by [?], arises from the jets deflection in the radial direction. Their direction of rotation thus depends on the relative position of the two opposed jets with respect to their axis of symmetry. At this stage of the analysis, it can be presumed that the formation of these vortices, which contribute to increase the interface between two opposed jets, promotes mixing. It is also observed that the interface between two opposed jets occupies most of the vacated dimension, i.e. the interface tends to spread along the radial direction in the form of a thin sheet. However, the combination of the local confinement with the uniform return flow via the porous plates imposes a reversal of this sheet-like structure, thus forming a large-scale vortical structure whose size is typically of order $L$, the mid-distance between the axis of two coplanar jets. When increasing the Reynolds number (not shown here), the area of impact of the jets appears more stable. The interface between two opposed jets seems to be more planar, reflecting the increased influence of the amount of axial compressive strain and radial stretching. As a result, vortices are less identifiable. Similarly, the extent of the interface seems to limit deflections in the radial direction.

Large scale structures occupying the entire width of the mixing layer surrounding the jets are also identifiable. These structures, stemming from the shear layer instabilities, are made visible by the engulfment of fluid of homogeneous concentration close to the overall average concentration within the vortices. The inlet velocity being substantially greater than the output velocity, vortices propagate to the average plane of stagnation. It is therefore expected that the stagnation region is the seat of many pairings of structures. The role of the oscillations of the jets on the pairing structures will be difficult to be evaluated, but their impact on the mixing process is most likely non-negligible.

This analysis serves to emphasize the complex nature of this flow: while jets-core regions typically exhibit levels of scalar concentration constant along the jets-axis, thus reflecting the low level of initial turbulence intensity, impingement regions in between two opposed-jets and mixing layers surrounding the jets accompanied by their instabilities require more attention to be paid to their own characteristics, closely linked to the influence of confinement.

\section{Scalar-variance budget along the axis of two confined-opposed-jets}

We aim here at giving a deeper look at the processes taking place in between two COJ by analysing the scalar-variance transport equation along the jets axis. We start with the advection-diffusion scalar transport equation

$$
\partial_{t} \zeta+u_{j} \partial_{j} \zeta=\kappa \partial_{j}^{2} \zeta
$$

where Einstein's summation convention is implied on the repeated indices, and $\kappa$ is the molecular diffusivity of the scalar quantity being passively transported. $\partial_{t} \equiv$ $\partial / \partial t, \partial_{j}$ denotes derivative with respect to $x_{j}$, and $\partial_{j}^{2}$ is the Laplacian $\partial^{2} / \partial x_{j} \partial x_{j}$. Subtracting the Reynolds-averaged counterpart of Eq. (??) to itself, multiplying the resulting transport equation of the fluctuating scalar concentration field by $\zeta$ (in the following, lower-case letters will represent fluctuating quantities), using continuity, and time-averaging, the one-point scalar-variance budget equation is 
obtained, as follows

$$
\underbrace{\partial_{t}\left\langle\zeta^{2}\right\rangle+U_{j} \partial_{j}\left\langle\zeta^{2}\right\rangle}_{T}=\underbrace{-2\left\langle\zeta u_{j}\right\rangle \partial_{j} Z}_{P}-\underbrace{\partial_{j}\left\langle u_{j} \zeta^{2}\right\rangle}_{F D}+\underbrace{\kappa \partial_{j}^{2}\left\langle\zeta^{2}\right\rangle}_{M D}-2\langle\chi\rangle,
$$

where capital letters denote first-order moments of the velocity components and the mean value of the scalar, and angular brackets symbolize time-averaging, i.e. $\langle\zeta\rangle=0$ and $\left\langle u_{j}\right\rangle=0$. In Eq. (??), the two terms in the left-hand-side account for the total (temporal and convective) variation of the energy of the fluctuating scalar concentration field, $T$. The first term in the right-hand-side of Eq. (??) accounts for the production from the interaction with the mean scalar concentration field, $P$, the second term is the diffusion through the fluctuating velocity field, $F D$, and the third term represents the diffusion of the energy of the scalar concentration field by its molecular diffusivity, $M D$. Finally, $\langle\chi\rangle=\kappa\left\langle\partial_{j} \zeta \partial_{j} \zeta\right\rangle$ is the mean scalar dissipation rate, i.e. the destruction of the variance through the molecular diffusivity effects.

Together with the axisymmetry hypothesis about the $y$ axis (with $r_{x} \| x$ ), Eq. (??) becomes

$$
\begin{array}{r}
\underbrace{U_{y} \partial_{y}\left\langle\zeta^{2}\right\rangle+U_{x} \partial_{r_{x}}\left\langle\zeta^{2}\right\rangle}_{T}=\underbrace{-2\left\langle u_{y} \zeta\right\rangle \partial_{y} Z-2\left\langle u_{x} \zeta\right\rangle \partial_{r_{x}} Z}_{P}- \\
\underbrace{\partial_{y}\left\langle u_{y} \zeta^{2}\right\rangle-\frac{1}{r_{x}} \partial_{r_{x}} r_{x}\left\langle u_{x} \zeta^{2}\right\rangle}_{F D}-2\langle\chi\rangle,
\end{array}
$$

where stationarity is assumed and the molecular diffusion term is considered to be negligible.

As a preliminary step of the analysis of each term present in Eq. (??), we choose to first discuss the distribution of the scalar variance in the COJ flow, as depicted in Fig. ??a. Some common features of the energy injection of the scalar fluctuations and of the axial component of the velocity field are to be emphasized. High level of axial velocity fluctuations, $u_{y}$, is created by the strong compressive strain of the axial velocity component in the impingement region. This strong compressive strain rate operates over very short distances, of the order of the interface which separates fluids with quasi-constant velocities. It leads to energy injection over a direction perpendicular to the instantaneous stagnation plane, thus not necessarily exactly parallel to $x$. However, this strong compressive strain is continuously transported along the axial direction through the action of the instabilities responsible for the oscillations of the interface. Therefore, the main impact of this oscillation process is that the strain of the mean velocity field operates over large length scales compared to the instantaneous interface. This can be observed on the velocity spectra which exhibit strong energy at scales representing the large scale variations of the mean velocity field [? ]. Therefore, the spatial gradients of the mean velocity field at some scale induces fluctuations at that scale. Most of the kinetic energy belongs to the axial component of the Reynolds stress tensor, $\left\langle u_{y}^{2}\right\rangle$ while less energy is given to $\left\langle u_{x}^{2}\right\rangle$. This results in a significant anisotropy level in this region. The same phenomenology is responsible for the injection of energy of the scalar fluctuations. The strong compressive strain of the quasi-unperturbed scalar field over the short interface width leads to production of intense fluctuations. Because of the oscillation process, this injection also operates over large distances compared to the instantaneous interface width. 


\subsection{Assessment of terms transport (T), production (P) and fluctuations diffusion (FD)}

All terms in Eq. (??) are evaluated from our experiments along the axis of two COJ, as shown in Fig. ??b, as functions of $y / d_{j}$. Some of the particularities of the kinematic structure of the flow translate in some peculiarities of the transport of energy. First, in the whole region dominated by the mean flow, i.e. in the unperturbed potential core of the jets, all terms of Eq. (??) can be disregarded because they remain nearly equal to zero. All the contributions to Eq. (??) take place in the central region of the chamber of width $2 \Delta$, i.e. in the region where the mean axial velocity decays to zero. In the following, we explain the behavior of all terms in Eq. (??).

Because the horizontal mean velocity, $U_{x}$, is uniformly zero along the jets axis, the only contribution to the term $T$ comes from its vertical component, $U_{y} \partial_{y}\left\langle\zeta^{2}\right\rangle$. In our arbitrary chosen frame of reference, where $y=0$ at the mean stagnation point, the mean axial velocity, $U_{y}$ is positive for $y<0$ and is negative for $y>0$, whereas the scalar-variance $\left\langle\zeta^{2}\right\rangle$ symmetrically decreases from its maximum at $y=0$ towards the jets injection points. Therefore, term $T$ is always positive, symmetric with respect to $y=0$, it is equal to zero for $y=0$ as well as out of the central region. The values of $T$ reported here correspond to an increase of the energy of the scalar fluctuations when one follows the mean vertical stream, whereas in the central region it is zero because of the stagnation.

The large-scale anisotropy of the flow and the scalar fields reflects in the production term, $P$ for which the maximum contribution is given by $-2\left\langle u_{y} \zeta\right\rangle \partial_{y} Z$, being thus associated with the axial gradient of the mean scalar field and the strongest level of vertical velocity fluctuations, $u_{y}$ compared to the radial velocity fluctuations, $u_{x}$. In our arbitrary chosen frame of reference, where $Z>0$ along the jets axis for $y>0$ and $Z<0$ for $y<0$, the mean scalar gradient, $\partial_{y} Z$ varies between zero in both regions of the jets injection and its peak takes a positive value for $y=0$. A blob of fluid moving with a positive vertical velocity fluctuation from the central region (rich in energy of the scalar fluctuations) through the injection region $y>0$ is associated with a negative scalar fluctuation, thus resulting in a scalar-velocity correlation term which is $\left\langle u_{y} \zeta\right\rangle<0$ in the central region and $\left\langle u_{y} \zeta\right\rangle=0$ near the injection regions. Therefore, the production term is equal to zero near the injection regions, but it is always positive and peaks to a large value near $y=0$.

The transport of energy of the fluctuating scalar field by the fluctuating velocity field, $F D$ mostly results from the contribution of $-\partial_{y}\left\langle u_{y} \zeta^{2}\right\rangle$. Since the maximum of the scalar variance $\left\langle\zeta^{2}\right\rangle$ is located at $y=0$, then term $\left\langle u_{y} \zeta^{2}\right\rangle$ is:

-positive for $y>0$, where a blob of fluid moving with a positive vertical velocity fluctuation necessarily moves from the central region (rich in energy of the scalar fluctuations) through the injection region $y>0$, which is poor in energy;

-negative for $y<0$ where only a negative velocity fluctuation may be associated with a transport of scalar variance from the rich, central region towards the poorer, peripheral region. In conclusion, term $\left\langle u_{y} \zeta^{2}\right\rangle$ varies between zero in both the region of the jets injection (no initial fluctuations) and for $y=0$, with positive values for $y>0$ and negative values for $y<0$. This behaviour of term $\left\langle u_{y} \zeta^{2}\right\rangle$ leads, after calculating its $y$-derivative and by changing its sign, to the variation of term $F D$ as illustrated on Fig. ??b, i.e. positive near the injection jets, and negative in the central region. This shape is fully consistent with the transport of the scalar variance from the rich central region, towards the poorer injection regions. 


\subsection{Scalar dissipation rate}

The full characterization of the scalar variance dissipation rate, $\chi(x, y)=$ $\kappa \nabla \zeta \nabla \zeta(x, y)$ requires to capture the local variations of the scalar concentration field through the simultaneous measurements of the three components of the mixturefraction gradient-vector, $\nabla \zeta$. A reliable estimate of $\chi(x, y)$ is very delicate to provide because passive scalar measurements generally suffer from under-resolution of the small scales of the fluctuating scalar field, but also the presence of noise affecting mainly the high wavenumbers. Moreover, since only the two in-plane components of the scalar gradient vector can be obtained from our PLIF measurements, direct differentiation of the raw scalar does not lead to reliable evaluation of the mean scalar dissipation rate. This statement is illustrated in Fig. ??b. The direct estimate of $\chi(x, y)$ from finite differences, noted $-\langle\chi\rangle_{\text {direct }}$ (solid line), is negligible with respect to the other terms and does not allow the one-point energy budget equation to be closed.

Therefore, some hypotheses are required to provide a reliable estimate of $\langle\chi\rangle$. In a previous study [?], an analysis of the scalar concentration field was carried in a partially stirred reactor (PASR) with the aim at providing an estimate as accurate as possible of the scalar variance dissipation rate. The statistical properties of the fluctuating scalar concentration field in the central zone of the reactor allowed to adopt the homogeneity assumption throughout the study. Different methods to estimate $\chi(x, y)$ were tested: the classical method based on the direct differentiation of the scalar field by finite-differences, the method based on the determination of the osculating parabola of the autocorrelation function, the method based on the extrapolation to small scales of the second-order structure functions and, a method based on a large-scale, one-point energy balance. A good agreement was found between each estimate when applied to the Wiener-filtered scalar concentration fields.

In the present case, another indirect method to estimate $\langle\chi\rangle$ is proposed. It is based on the scalar-variance budget evaluated on the axis of two opposed-jets, Eq. (??). The justification is the following: the axisymmetry assumption under which Eq. (??) is established along the axis of two opposed-jets is well verified, and all the other terms present in this budget involve mean quantities which are well resolved by our experimental apparatus. The mean scalar variance dissipation rate may thus be determined along the axis of two opposed-jets, $\chi(x=0, y)$ as the closing term of Eq. (??). This is noted $-\langle\chi\rangle_{\text {budget }}$ and is represented in Fig. ?? with dotted line.

We have thus reliably determined the distribution along the common axis of the COJ flow of $-\langle\chi\rangle$. We are now interested in relating the high values of $-\langle\chi\rangle$ in the central region of the flow, to the local instantaneous aspect of flow and mixing. Figure ??(a-c) shows typical distributions of the fluctuating scalar concentration fields, $\zeta(x, y)$, the instantaneous scalar variance dissipation fields, $\chi(x, y)$ and logarithm of the instantaneous scalar variance dissipation fields, $\log \chi(x, y)$ which allow for the low dissipation values structures to be revealed, for one pair of opposed-jets as a function of $x / d_{j}$ and $y / d_{j}$. The jet centreline coincides with $x / d_{j}=0$. Some basic features of the structural organization of the $S c \approx 1$ conserved scalar mixing in a turbulent flow are revealed with these typical maps and discussed in the following.

The conserved scalar dissipation rate fields generated by the confined-opposedjets flow are mostly organized as sheet-like strained laminar diffusion layers, as previously evidenced by [?] in turbulent shear flows. We make the same arguments as these authors: if line-like topologies were to be present instead of sheet-like topologies, then the intersection of the measurement plane with these linear structures would produce roughly (some) circular intersections. Moreover, 
since the confined-opposed-jets flow is statistically axisymmetric around the jetsaxis, one could expect a high probability of presence of these topologies if they were to exist. Yet, no such topology is observable here, neither in the linear nor in the logarithmic representations of the dissipation rate fields.

The linear representation serves to point out that isolated layers of high dissipation values are mainly present at the interface in between two confined-opposed-jets or, at the interface of the injecting jet with the return flow. As a consequence, these layers present a preferential orientation. At the interface between the injecting jet and the return flow, they are mostly oriented at $45^{\circ}$ with respect to the jet-axis. In this region, i.e. the shear layers, it was shown that the mean vorticity matches the mean shear, the latter being oriented at $45^{\circ}$ [? ]. The dissipation layers at the interface between two opposed-jets have a random orientation and a larger degree of distortion. On the other hand, interacting layers of low dissipation values preferentially populates the central region located in between two pairs of opposed-jets, as revealed by the logarithmic presentations. They are generally oriented very differently from adjacent layers, and the scale over which they are distorted is typically a few layer thickness. This is a classical result for $S c \approx 1$ conserved scalar mixing for which the scalar diffusion scale, $\lambda_{D}$ is comparable to the vorticity diffusion scale, $\lambda_{v}$. As a consequence, the dissipation layers are distorted by the differential rotation effects due to gradients in the vorticity field. Two adjacent layers may be subject to significantly different vorticity and strain rate values, leading to their different orientations and thickness [?].

\section{Scale-by-scale scalar-variance transport equation in the axisymmetric context}

\subsection{Scale-by-scale scalar-variance budget equation}

Using the same procedure as outlined in [? ? ? ], we start with the advectiondiffusion scalar equation written, within the Reynolds decomposition framework, at the two points $\underline{x}$ and $\underline{x}^{+}$separated by a scale $\underline{r}=\underline{x}^{+}-\underline{x}$,

$$
\begin{gathered}
\partial_{t} \zeta+U_{j} \partial_{j} Z+U_{j} \partial_{j} \zeta+u_{j} \partial_{j} Z+u_{j} \partial_{j} \zeta=\kappa \partial_{j}^{2}(Z+\zeta) \\
\partial_{t} \zeta^{+}+U_{j}^{+} \partial_{j}^{+} Z^{+}+U_{j}^{+} \partial_{j}^{+} \zeta^{+}+u_{j}^{+} \partial_{j}^{+} Z^{+}+u_{j}^{+} \partial_{j}^{+} \zeta^{+}=\kappa \partial_{j}^{+2}\left(Z^{+}+\zeta^{+}\right),
\end{gathered}
$$

where Einstein's summation convention is implied. Capital letters denote mean quantities which are considered as being stationary, $\zeta$ and $u_{j}$ are the fluctuating scalar concentration and velocity fields respectively, i.e. $\langle\zeta\rangle=0$ and $\left\langle u_{j}\right\rangle=0$ and $\kappa$ is the molecular diffusivity. The superscript ${ }^{+}$refers to quantities written at $\underline{x}^{+}$. In Eqs. (??) and (??), $\partial_{t} \equiv \partial / \partial t, \partial_{j}$ and $\partial_{j}^{+}$denote derivatives with respect to $x_{j}$ and $x_{j}^{+}$respectively, and $\partial_{j}^{2}$ is the Laplacian $\partial^{2} / \partial x_{j} \partial x_{j}$.

Subtraction of Eq. (??) from Eq. (??) yields a transport equation for the fluctuating scalar increment, $\delta \zeta=\zeta^{+}-\zeta$, viz.

$$
\begin{array}{r}
\partial_{t} \delta \zeta+\delta\left(U_{j} \partial_{j} Z\right)+\delta\left(u_{j} \partial_{j} Z\right)+\delta\left(U_{j} \partial_{j} \zeta\right)+u_{j}^{+} \partial_{j}^{+} \delta \zeta+u_{j} \partial_{j} \delta \zeta= \\
\kappa\left(\partial_{j}^{2}+\partial_{j}^{+2}\right)(\delta(\zeta+Z)),
\end{array}
$$

where increments of any function $f$ are defined as $\delta f=f^{+}-f$. Eq. (??) is obtained with the assumption that points separated by any distance $\underline{r}$ are independent, i.e. 
any functions $f$ and $f^{+}$depend only on $\underline{x}$ and $\underline{x}^{+}$respectively.

Multiplying Eq. (??) by $2 \delta \zeta$ and averaging, one can easily obtain the two-point scalar-variance transport equation

$$
\begin{gathered}
\partial_{t}\left\langle(\delta \zeta)^{2}\right\rangle+2\left\langle\delta\left(U_{j} \partial_{j} \zeta\right) \cdot \delta \zeta\right\rangle+2\left\langle\delta\left(u_{j} \partial_{j} Z\right) \cdot \delta \zeta\right\rangle+2\left\langle\left(u_{j}^{+} \partial_{j}^{+} \delta \zeta+u_{j} \partial_{j} \delta \zeta\right) \cdot \delta \zeta\right\rangle \\
=2 \kappa\left\langle\left(\partial_{j}^{2}+\partial_{j}^{+2}\right) \delta \zeta \cdot \delta \zeta\right\rangle
\end{gathered}
$$

Following the approach proposed in [?], the gradient with respect to the midpoint is considered, i.e. $\underline{X}=1 / 2\left(\underline{x}+\underline{x}^{+}\right)$, resulting in

$$
\begin{gathered}
\partial_{j} \equiv-\frac{\partial}{\partial r_{j}}+\frac{1}{2} \frac{\partial}{\partial X_{j}} \\
\partial_{j}^{+} \equiv \frac{\partial}{\partial r_{j}}+\frac{1}{2} \frac{\partial}{\partial X_{j}} .
\end{gathered}
$$

Thus, $\partial_{X_{j}}=\partial_{j}+\partial_{j}^{+}$. Equation (??) can then be rearranged as

$$
\begin{gathered}
\partial_{t}\left\langle(\delta \zeta)^{2}\right\rangle+2\left\langle\delta\left(U_{j} \partial_{j} \zeta\right) \cdot \delta \zeta\right\rangle+2\left\langle\delta\left(u_{j} \partial_{j} Z\right) \cdot \delta \zeta\right\rangle+\left\langle\left(u_{j}^{+}+u_{j}\right) \partial_{X_{j}}(\delta \zeta)^{2}\right\rangle+2 \partial_{r_{j}}\left\langle\delta u_{j}(\delta \zeta)^{2}\right\rangle \\
=\kappa \frac{\partial^{2}}{\partial r_{j} \partial r_{j}}\left\langle(\delta \zeta)^{2}\right\rangle-2\left(\langle\chi\rangle+\langle\chi\rangle^{+}\right)
\end{gathered}
$$

Note that each term of Eq. (??) depends only on the scale separation vector $\underline{r}$, and of the point $\underline{x}$ at which Eq. (??) is assessed.

\subsection{Axisymmetric context}

An axisymmetric treatment is to be further applied to Eq. (??) in analogy with the treatment applied to the scale-by-scale kinetic energy budget in [?]. Axisymmetry of both the flow and scalar concentration field is assumed with respect to a direction specified by the unit vector $\underline{n}$, i.e. statistics are invariant to rotations in planes normal to $\underline{n}$ and symmetric with respect to $\underline{n}$. Note that in this context, large-scale inhomogeneous variations might be observed along the axial direction $\underline{n}$.

Within the underlying assumptions, each term of Eq. (??) which originally depends on the scale separation vector $\underline{r}$ is then made dependent on the scalar variables $r$ and $\mu$ only, defined by the following relations

$$
r^{2}=\underline{r} \cdot \underline{r} \text { and } r \mu=\underline{r} \cdot \underline{n}
$$

In a similar manner as [?] we then write

$$
\left\langle\delta u_{j}(\delta \zeta)^{2}\right\rangle=M(r, \mu) \cdot r_{j}+N(r, \mu) \cdot n_{j},
$$

where $M(r, \mu)$ and $N(r, \mu)$ are scalars which can be determined from planar measurements, providing that the axial direction $\underline{n}$ belongs to the measurement plane. 
This leads to the experimentally determinable scalar functions

$$
\begin{array}{r}
M(r, \mu)=\frac{\left\langle\delta u_{\perp}(\delta \zeta)^{2}\right\rangle}{r \sqrt{1-\mu^{2}}} ; \\
N(r, \mu)=\left\langle\delta u_{\|}(\delta \zeta)^{2}\right\rangle-\left\langle\delta u_{\perp}(\delta \zeta)^{2}\right\rangle \frac{\mu}{\sqrt{1-\mu^{2}}},
\end{array}
$$

where $u_{\|}$and $u_{\perp}$ are the velocity fluctuations parallel and perpendicular to $\underline{n}$ respectively. Therefore, in the axisymmetric context, $\partial_{r_{j}}\left\langle\delta u_{j}(\delta \zeta)^{2}\right\rangle$ becomes

$$
\partial_{r_{j}}\left\langle\delta u_{j}(\delta \zeta)^{2}\right\rangle \equiv\left(r \frac{\partial}{\partial r}+3\right) M(r, \mu)+\left(\mu \frac{\partial}{\partial r}+\frac{1-\mu^{2}}{r} \frac{\partial}{\partial \mu}\right) N(r, \mu) .
$$

For mathematical simplification purpose, we then introduce the scalar function $V$ such that $N(r, \mu)=\left(2+r \frac{\partial}{\partial r}\right) V$, with $V=\frac{1}{r^{2}} \int_{0}^{r} s N(r, \mu) d s$, where $s$ is a dummy variable. We further define

$$
N^{*}=\left(\mu \frac{\partial}{\partial r}+\frac{1-\mu^{2}}{r} \frac{\partial}{\partial \mu}\right) V
$$

and noting [?]

$$
\left(\mu \frac{\partial}{\partial r}+\frac{1-\mu^{2}}{r} \frac{\partial}{\partial \mu}\right)\left(r \frac{\partial}{\partial r}+2\right) V=\left(r \frac{\partial}{\partial r}+3\right)\left(\mu \frac{\partial}{\partial r}+\frac{1-\mu^{2}}{r} \frac{\partial}{\partial \mu}\right) V,
$$

Equation (??) becomes

$$
\begin{gathered}
\partial_{t}\left\langle(\delta \zeta)^{2}\right\rangle+2\left\langle\delta\left(U_{j} \partial_{j} z\right) \cdot \delta \zeta\right\rangle+2\left\langle\delta\left(u_{j} \partial_{j} Z\right) \cdot \delta \zeta\right\rangle+\left\langle\left(u_{j}^{+}+u_{j}\right) \partial_{X_{j}}(\delta \zeta)^{2}\right\rangle+ \\
2\left(r \frac{\partial}{\partial r}+3\right)\left(M(r, \mu)+N^{*}(r, \mu)\right)=\kappa \nabla^{2}(r, \mu)\left\langle(\delta \zeta)^{2}\right\rangle-2\left(\langle\chi\rangle+\langle\chi\rangle^{+}\right),(25)
\end{gathered}
$$

where the axisymmetric form of the Laplacian $\nabla^{2}(r, \mu)$ was introduced [?]. Therefore, Eq. (??) formally writes

$$
\begin{gathered}
D(r, \mu)+T(r, \mu)+P(r, \mu)+F D(r, \mu)+2\left(r \frac{\partial}{\partial r}+3\right)\left(M(r, \mu)+N^{*}(r, \mu)\right)= \\
\kappa \nabla^{2}(r, \mu)\left\langle(\delta \zeta)^{2}\right\rangle-2\left(\langle\chi\rangle+\langle\chi\rangle^{+}\right)
\end{gathered}
$$

where terms $D, T, P$, and $F D$ are the decay, transport, production and diffusion through fluctuations terms respectively.

After integration of Eq. (??) with respect to $r$, the final axisymmetric form of the scale-by-scale scalar-variance budget equation is

$$
\begin{array}{r}
G(r, \mu)=-\frac{1}{r^{3}} \int_{0}^{r} s^{2}(D(r, \mu)+T(r, \mu)+P(r, \mu)+F D(r, \mu)) d s+ \\
\kappa \frac{1}{r^{3}} \int_{0}^{r} s^{2} \nabla^{2}(r, \mu)\left\langle(\delta \zeta)^{2}\right\rangle d s-\frac{2}{3}\left(\langle\chi\rangle+\langle\chi\rangle^{+}\right),
\end{array}
$$

where $G=M(r, \mu)+N^{*}(r, \mu)$. 
In the following, the reduced form of this equation is investigated

$$
G(r, \mu)=-\frac{2}{3}\left(\langle\chi\rangle+\langle\chi\rangle^{+}\right)
$$

which comes to study the energy of the scalar fluctuations transferred at each scale by the velocity fluctuations.

\subsection{Results}

This section is devoted to the experimental investigation of Eq. (??).

\subsubsection{Scalar variance distribution at each scale in the impingement region}

The structure functions of any order discussed in the following are calculated by fixing the origin of the Cartesian coordinate system $\left(r_{x}, r_{y}\right)$ at the intersection of the $y$-axis of two opposed jets with the plane where the mean axial velocity is zero, therefore scalar and velocity increments, are calculated as $\delta \zeta \equiv \zeta\left(r_{x}, r_{y}\right)-$ $\zeta\left(r_{x}=0, r_{y}=0\right)$ and $\delta u_{i} \equiv u_{i}\left(r_{x}, r_{y}\right)-u_{i}\left(r_{x}=0, r_{y}=0\right)$ respectively.

The distribution of energy of the scalar fluctuations at each scale, $\left\langle(\delta \zeta)^{2}\right\rangle$ are presented as a function of the Cartesian coordinates $\left(r_{x}, r_{y}\right)$, in Fig. ??a and as a function of the cylindrical coordinates $(r, \mu)$, in Fig. ??b. The $y$-axis $\underline{n}$ is parallel to $y, r_{x}$ and $r_{y}$ are the separations in the $x$ and $y$ directions, therefore $r=\sqrt{r_{x}^{2}+r_{y}^{2}}$ and $\mu=\cos \left(\arctan \left(r_{x} / r_{y}\right)\right)$. Variations of energy at each scale are to be correlated with the distribution of the scalar variance, as represented in Fig. ??b, i.e. $\left\langle(\delta \zeta)^{2}\right\rangle$ is zero at the point $r_{x}=0, r_{y}=0$, then it increases much less in the vertical direction $r_{y}(\equiv \mu= \pm 1)$ than in the radial direction $r_{x}(\equiv \mu=0)$.

\subsubsection{Anisotropic scalar energy transfer in the impingement region of the COJ flow}

The energy of the scalar fluctuations transferred by the velocity fluctuations at a given scale $r$ and a spatial position $(r, \mu)$, which is equivalent to $\left\langle\delta u(\delta \zeta)^{2}\right\rangle$ in isotropic turbulence, writes as term $G$ in the present axisymmetric formalism. The distribution of this term for one pair of opposed-jets is illustrated in Fig. ??, for two particular directions: $\mu= \pm 1$ and $\mu=0$, i.e. the axial direction and the radial direction respectively.

For $\mu= \pm 1$, term $G$ is first positive for scales smaller than $\approx \Delta$ and then becomes negative for scales larger than $\approx \Delta$. We recall here that $\Delta$ is the characteristic length-scale over which the instantaneous interface between two opposed-jets is transported (mainly in the vertical direction). The positive values of term $G$ below $\Delta$ therefore does not signify an inverse energy cascade mechanism, it is rather the signature of the transport of energy produced at small scales by the largescales velocity fluctuations. The negative value of term $G$ for scales larger than $\Delta$ corresponding to a direct cascade reflects that the transport of energy by the turbulent fluctuations becomes dominant in this range of scales. It is to be noted that the transport of energy along the $y$-axis vanishes for scales larger than $\approx 1.6 d_{j}$ because of the absence of any fluctuations in this unperturbed flow region.

For $\mu=0$, term $G$ presents a similar evolution to that discussed above, i.e. first positive and then negative, although on a different range of scales and with significantly larger values. Again, the positiveness of term $G$ for scales smaller than $\approx 1.6 d_{j}$ does not signify an inverse energy cascade, it is rather the signature of large-scale inhomogeneity.

We propose a mechanism for this particular aspect. It was already underlined 
that in this flow, the origin of the energy of the fluctuations results from two contributions: the classical production mechanism which, however in the present case results from normal stresses acting on the scalar field instead of the traditional picture emanating from shear-stress dominated flow; and the large scale convective transport towards larger-scales due to the characteristic instabilities of this opposed-jets flow. Once these large-scale energy containing structures (illustrated in Fig. ??d) are generated, they escape the impingement region in the radial direction. It is to be noted that the homogeneous repartition of this energy in the radial direction is rarely observed, because of the quasi-systematic deviation of the opposed-jets with respect to their mean axis. Again, through this large-scale inhomogeneous process, energy is thus transferred to larger scales. This transfer is ultimately limited in the radial direction by the confinement, whose influence depends on the relative instantaneous position of each pair of opposed-jets neighbouring the pair of opposed-jets under study.

The right-hand side term of Eq. (??), i.e. the distribution of the two-points scalarvariance dissipation rate $\langle\chi\rangle+\langle\chi\rangle^{+}$multiplied by $2 / 3$ is also depicted in Fig. ??, for the same particular directions. This term is clearly preferentially oriented towards the axis $\mu=0$, corresponding to the radial direction $r_{x}$. This has to be correlated to the one-point scalar variance dissipation rate, as shown on Fig. ??, which revealed dissipation structures mostly present at the interface of two opposed-jets with the shape of sheet-like strained laminar diffusion layers. Note that mean values of the scalar dissipation rate were determined using the method described in [? ]. For case A, the maximum difference between estimated values of $\langle\chi\rangle$ was less than $10 \%$. For the whole range of the investigated cases (A to F), the maximum difference was of $27 \%$.

The reduced form of the scalar variance budget, as given by Eq. (??), is not at all equilibrated, whatever the direction. Although the energy transfer is intensified along the radial direction $(\mu=0)$, term $\mathrm{G}$ remains much less important than the total energy transferred. The unbalance between the LHS and RHS terms of Eq. (??) may be explained by the absence of the molecular diffusion term at small scales. At intermediate and large scales, large scale processes, not accounted in this budget, are expected to dominate the total energy transfer.

A more complete characterization of the mixing in between two closely-spaced COJ is provided with the evaluation of the reduced form of the scalar variance budget, as given by Eq. (??), when the Reynolds number is varied. The impact of the geometry, through the ratio $h / d_{j}$ is also discussed in the following.

It is illustrated in Fig. ?? that, either for the axisymmetric direction $(\mu= \pm 1)$ or the radial direction $(\mu=0)$, the energy of the scalar fluctuations transferred by the velocity fluctuations at a given scale is enhanced when the Reynolds number is increased. This statement is true for both geometries. However, the energy balance as given by Eq. (??) remains unclosed, as the energy to be transferred is also increased in the same proportions. This is evidenced by term $G /\left(\langle\chi\rangle+\left\langle\chi^{+}\right\rangle\right)$, i.e. the energy of the scalar fluctuations normalized by the total amount of energy at a given scale, which is expected to reach the asymptotic value $-2 / 3$ as the Reynolds number is large. In this flow, this term remains unchanged at a given scale over the Reynolds numbers range investigated, for a given geometry.

It is interesting to note that along the axisymmetric direction $(\mu= \pm 1$, Fig. ??a), any impact of the geometry on the normalized transfer terms are undistinguishable when the scales over which term $G /\left(\langle\chi\rangle+\left\langle\chi^{+}\right\rangle\right)$is evaluated are normalized by the inlet diameters, i.e. $r / d_{j}\left(d_{j}=10 \mathrm{~mm}\right.$ for cases A and D, $d_{j}=6 \mathrm{~mm}$ for cases $\mathrm{C}$ and $\mathrm{G}$ ). In particular, the location in the normalized scale range of the maximum positive values, the change of sign and the minimum negative values remain the 
same. On the other hand, when the normalized term $G /\left(\langle\chi\rangle+\left\langle\chi^{+}\right\rangle\right)$is evaluated over the normalized scale range $r / d_{j}$ along the radial direction $(\mu=0$, Fig. ??b), the impact of the geometry is clearly revealed at the largest scales. Although the evolution of term $\mathrm{G}$ remains self-similar over the scales for a given geometry, the significant difference of its evolutions at large scales further illustrates that the flow developing from the stagnation region is no more controlled by the geometry and is related to large-scale instabilities.

\section{Conclusions}

We have investigated the mixing of a conserved passive scalar for $S c=1$ ( $S c$ is the Schmidt number) in axisymmetric turbulence for which the initial injections of turbulent kinetic energy and scalar variance are similar. Two confined-opposed-jets (COJ) were experimentally studied through simultaneous PIV and PLIF measurements, for several flow regimes. One-point transport equation for the scalar variance was assessed through experimental data, along the common axis of the two opposed jets, and different physical phenomena were revealed (production, diffusion, dissipation). In the mean stagnation plane, here identified as $y=0$, we have shown that:

-the transport through the mean vertical velocity is zero;

-the production term $P$ is positive and maximum. The latter is due to its proportionality to the gradient of the mean scalar field;

-the diffusion through fluctuations $F D$ is negative. The production of scalar variance is equilibrated by term $F D(\sim 75 \%)$ and the mean dissipation of the scalar variance $\sim 25 \%$.

To further assess the scalar behaviour at each scale in this anisotropic, but axisymmetric, flow, a scale-by-scale scalar variance budget equation was derived for axisymmetric turbulence. This equation reduces to Yaglom's 4/3 law, under additional restrictions. The equation was assessed through experimental data, in the impingement region between the two confined-opposed-jets. In particular, the anisotropic energy transfer along different directions was quantified. We have shown that for scales smaller than the size of the central region, $\Delta$, the cascade of the scalar variance is completely inhibited, independently of the particular direction. For scales larger than $\Delta$, the apparent aspect of the energy transfer is that of an inverse energy cascade. Nonetheless, inhomogeneity of the flow at those scales is directly responsible for these positive values. This behaviour of the scalar strongly contrasts with respect to the kinetic energy cascade, for which it was emphasized [? ] that the cascade was classical and direct in the plane perpendicular to the common axis of the two opposed jets, whereas it was strongly inhibited along the axial direction. The difference between the cascade of the scalar variance and that of the kinetic energy stands in morphological differences between the two fields. Although the two of them are injected at the same scale, the velocity field is nourished in energy from the stagnation points between different pairs of opposed jets, whereas the scalar field is completely mixed in the central plane. Before exiting the chamber, the already mixed fluid only meets another well-mixed fluid. Further efforts are required to assess the other terms involved in the energy budget equation at each scale, particularly from numerical simulations which are better adapted to properly determine small-scale statistics for both velocity and scalar fields. 
Financial support from the French National Research Agency, under the projects
ANISO' and 'MUVAR' is gratefully acknowledged. Professor P.E. Dimotakis is

Financial support from the French National Research Agency, under the projects
'ANISO' and 'MUVAR' is gratefully acknowledged. Professor P.E. Dimotakis is warmly thanked for his contribution to the initiation of this study. 


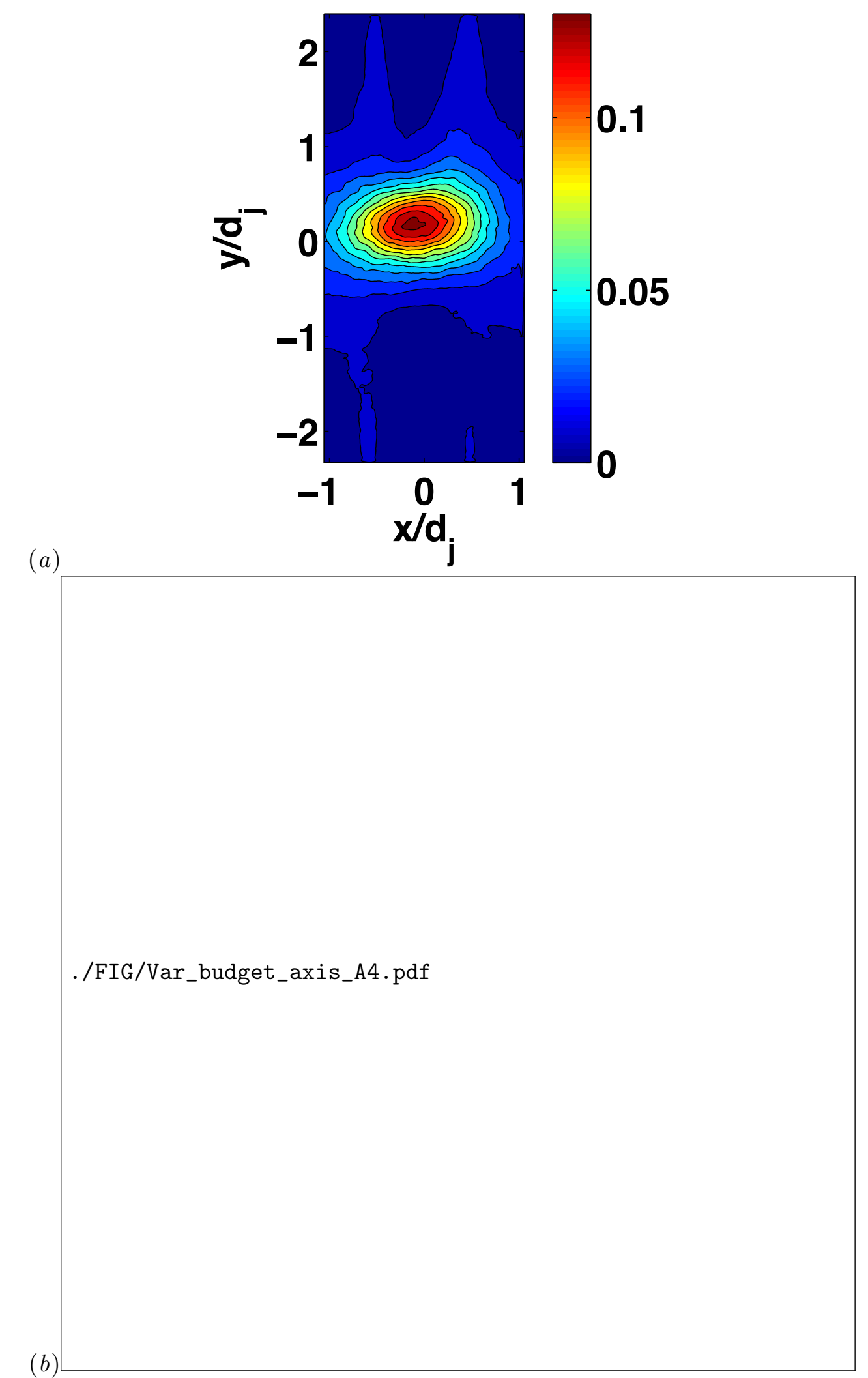

Figure 3. (a): Contour plots of the scalar variance, $\left\langle\zeta^{2}\right\rangle$ as a function of $x / d_{j}$ and $y / d_{j}$. Contour plots in increments of 0.01 and bounded by 0 and 0.13 ; (b): Scalar-variance budget (multiplied by $d_{j} / V_{i n j}$ ) on the axis of two confined opposed jets, as given by Eq. (??). (--): $T ;(+): P ;(\circ): F D ;$ solid line: $-\langle\chi\rangle_{\text {direct }}$. dotted line: $-\langle\chi\rangle_{\text {budget }}$, obtained as the closing term of the budget Eq. (??). Case A.

(a) 
$(a)$

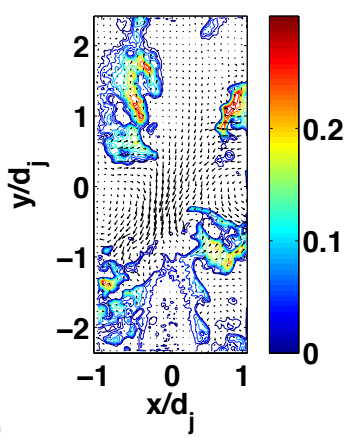

(b)
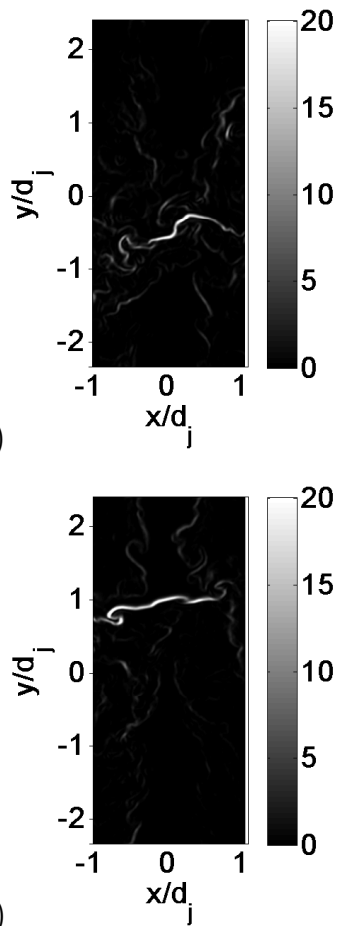

$(c)$

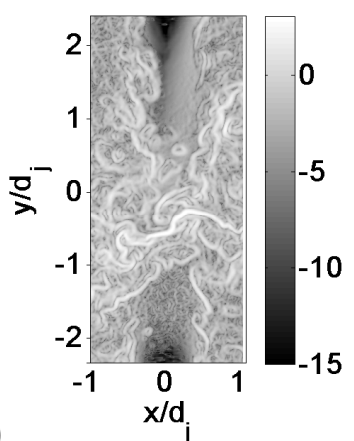

$(f)$

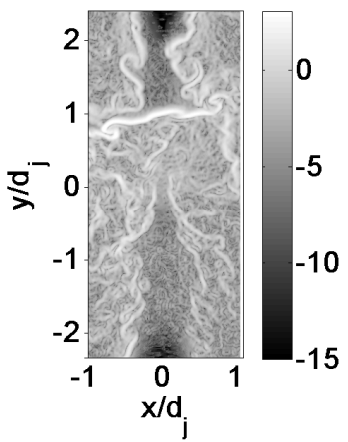

Figure 4. (a) and (d): Two examples of contour plots of the fluctuating scalar concentration field, $\zeta(x, y)$ and fluctuating velocity vectors field, 1 of every 10 vectors displayed; (b) and(e): Two examples of instantaneous scalar dissipation rate fields, $\chi(x, y) ;(c)$ and (f): Two examples of logarithm of instantaneous scalar dissipation rate fields, $\log \chi(x, y)$. Case A. 
$(a)$
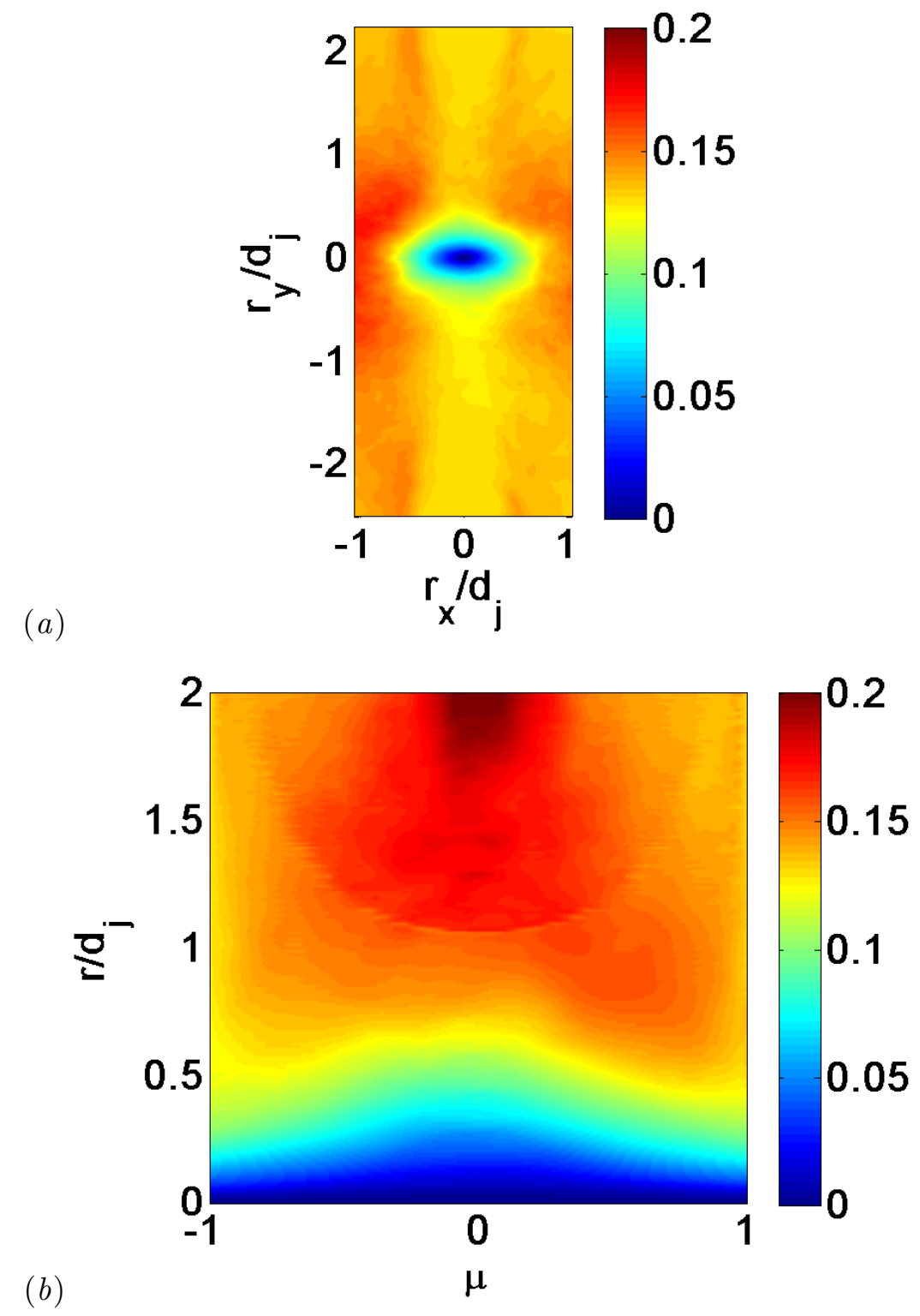

Figure 5. Distribution of the scalar variance at each scale. $(\mathrm{a}):\left\langle(\delta \zeta)^{2}\right\rangle\left(r_{x}, r_{y}\right) ;(\mathrm{b}):\left\langle(\delta \zeta)^{2}\right\rangle(r, \mu)$. Case A.

Figure 6. Terms in Eq. (??) for different values of $\mu$ : $\mu= \pm 1$ ( $\square$ : LHS, solid line: RHS); $\mu=0$ (--: LHS, $\times$ : RHS). Case A.

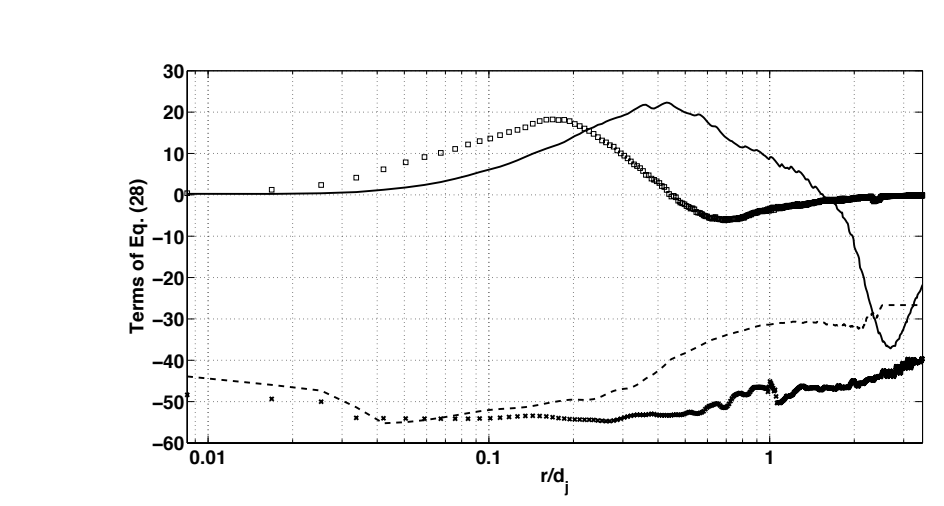



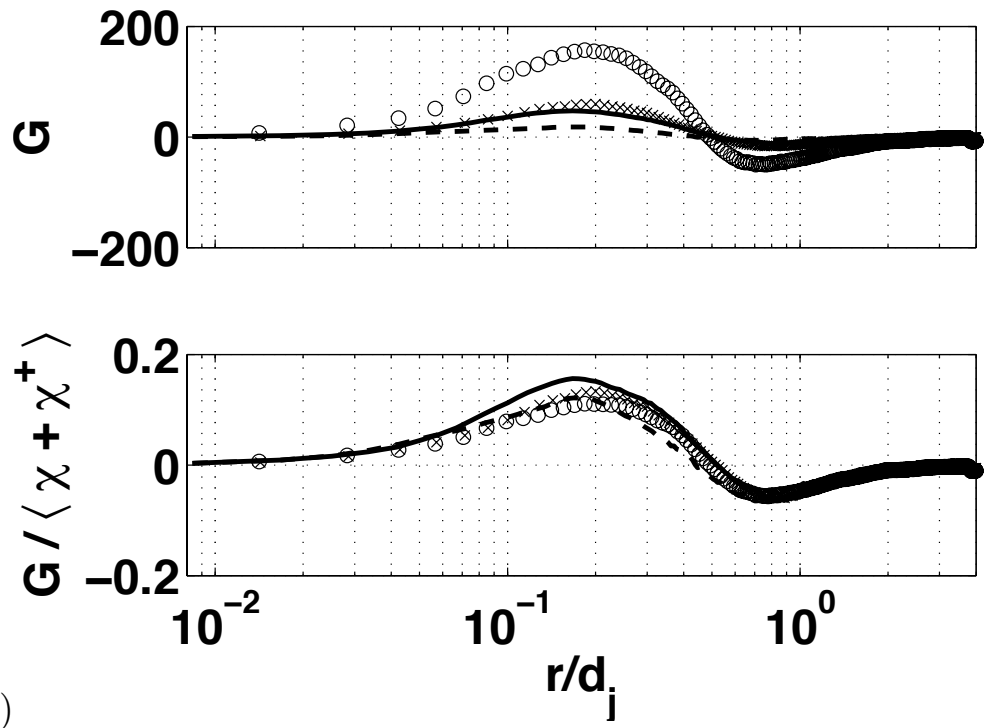

$(a)$
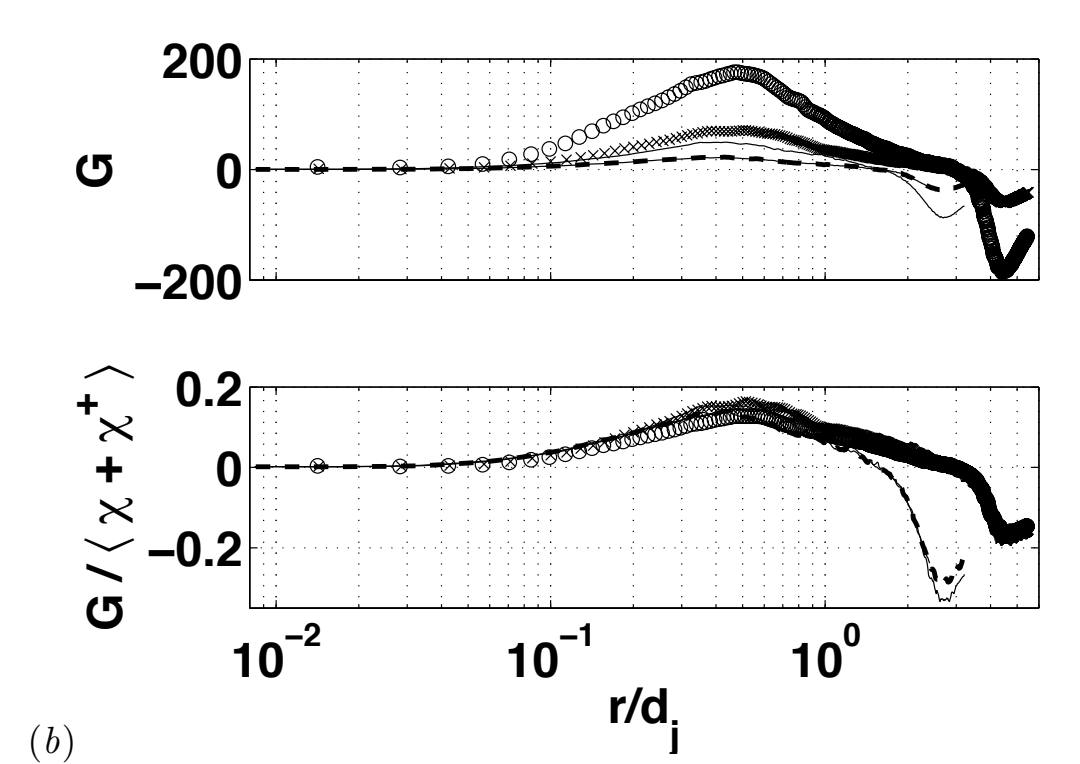

Figure 7. Top: term $G$ of Eq. (??); bottom: term $G$ normalized by the two points scalar variance dissipation rate, $G /\left(\langle\chi\rangle+\left\langle\chi^{+}\right\rangle\right)$. (a): $\mu= \pm 1 ;(\mathrm{b}): \mu=0$. $(--)$ : case $\mathrm{A} ;(\times)$ : case $\mathrm{C} ;(-)$ : case $\mathrm{D} ;(0)$ : case G. 